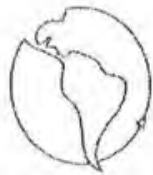

\title{
Exclusión, Poder y Relaciones Sociales
}

Daniel Duhart Programa de Magíster en Antropología y Desarrollo, Universidad de Chile. dduhart@gmail.com

NA AOISYER EN ANTROPOLOGIA Y DESARBOLLO

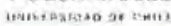

\section{Introducción}

El concepto de exclusión social, así como el de marginación' ${ }^{1}$, han sido utilizados desde algunos años en el discurso público y académico ${ }^{2}$ para referirse a una serie de situaciones de privación material, cultural y política. Ya sea la preocupación por la superación de la pobreza, la situación de los pueblos indígenas, o la realidad de poblaciones invisibles a las políticas sociales, tales como los ancianos, las mujeres y la juventud, múltiples han sido los casos en que se ha hecho referencia a estos conceptos. Sin embargo, al momento de hablar sobre la exclusión social y diseñar políticas para superarla e integrar a estos grupos, entran en juego una serie de reflexiones más profundas acerca de las implicancias y supuestos implícitos en este proceso.

Por un lado, desde una perspectiva más conceptual, ¿qué luces nos puede dar el concepto de exclusión social acerca de la naturaleza misma de la privación?, ¿qué nuevo nos entrega en relación a otros conceptos, como el de pobreza? Y en este sentido, ¿qué aporte puede hacer para la acción y el diseño de políticas de inclusión? Por otro lado, como extensión de esta reflexión, y desde una perspectiva más amplia del desarrollo, ¿para qué queremos integrar a estos grupos sociales excluidos del sistema económico-

\footnotetext{
I En América Latina el concepto de marginación social fue más utilizado en un comienzo, el cual presentaba un énfasis más bien estructural en su análisis de la realidad social, generándose un muy rico debate a fines de los años sesenta y comienzos de los años setenta (ver las obras de Aníbal Quijano, Roger Vekemans, Fernando Henrique Cardoso y José Nun, por ejemplo). A comienzos de los años noventa el concepto de exclusión social fue incorporado en el debate $y$ análisis, el cual provenía desde Europa y presentaba una mirada más centrada en las relaciones sociales. A pesar de las diferencias de énfasis entre ambos conceptos, en este artículo sc intenta combinar un enfoque de actores con una mirada estructural al momento de reflexionar sobre las implicancias de la exclusión y la marginación sociales.
}

2 Bengoa, José. "La exclusión", en La comunidad perdida, Ediciones Sur, 1996, Santiago. 
político y social?, ¿para que ellos cambien y adopten los cánones del sistema?, ¿o no será que el sistema mismo es inherentemente excluyente? Y en este sentido, ¿no será también necesario un proceso de transformación del mismo sistema durante este proceso de inclusión o integración social?; ;cómo se puede lograr este proceso paralelo e interconectado? Estas son sólo algunas de las reflexiones que consideramos vitales al momento de reflexionar sobre el concepto de exclusión social, así como sobre las políticas sociales en el proceso de desarrollo general, y que pretendemos abordar en el presente artículo.

\section{¿Qué es Exclusión Social?}

Como hemos dicho, existe una amplia discusión y debate sobre el concepto de exclusión social, su aporte, sus usos, y sus implicancias para la política social. Uno de los más importantes aportes es el realizado por el economista Nobel Amartya Sen, en el contexto de su enfoque de pobreza como privación de capacidades. Para el caso de América Latina, la CEPAL ha abordado el concepto y lo ha incluido en sus reflexiones sobre la superación de la pobreza.

De acuerdo con Amartya Sen, el origen del concepto se remonta al ańo 1974, en Europa, cuando el entonces Secrétaire d'Etat a l'Action Sociale del Gobierno Francés, René Lenoir, se refirió a la población marginal y "socialmente desadaptada" que había quedado excluida del Estado Benefactor, un $10 \%$ de la población en ese momento. Al describir a este sector, los caracterizaba como los "discapacitados, suicidas, ancianos, niños abusados, drogadictos, delincuentes, padres-madres solteros, hogares con problemas múltiples, personas marginales, etc.". 3

De acuerdo con Amartya Sen, en los años siguientes el concepto de exclusión social comenzó a ser utilizado en la discusión académica y pública para referirse a una amplia gama de situaciones, convirtiéndose en un concepto "paraguas" que abarca todo, pero que no quiere decir nada. Las

' Sen, Amartya, "Social Exclusion: concept, application and scrutiny", Office of Envirionment and Social Development, Asian Development Bank, June 2000, página 1. 
cosas de las cuales las personas pueden ser "excluidas" serían: "un sustento; empleo seguro y permanente; ingresos; propiedad, crédito y tierra; niveles minimos de consumo; educación, destrezas y capital cultural; del Estado Benefactor; ciudadanía y equidad legal; participación democrática; bienes públicos; de la nación o raza dominante; de la familia y sociabilidad; de la humanidad, respeto, realización personal y entendimiento. " $\mathrm{O}$ sea finalmente de todo, entonces: ¿cuál es el aporte del concepto?

En América Latina el concepto también comenzó a ser utilizado con bastante fuerza en los años noventa, en el contexto del retorno de la democracia y el lanzamiento de una nueva generación de políticas sociales para la superación de la pobreza. De acuerdo con la CEPAL,

"... la exclusión social es un proceso que surge a partir de un debilitamiento progresivo o un quiebre duradero de los lazos que unen a los sujetos con la sociedad a la que pertenecen, de tal modo, que se establece una división entre los que están dentro y quienes están fuera de ella. Por lo tanto, la exclusión alude a procesos a través de los cuales algunas personas no sólo poseen menos, sino que son crecientemente incapaces de acceso a los diferentes ámbitos de la vida social."

Luego agrega:

"No es por tanto, solamente un problema de desigualdad y pobreza, sino un fenómeno basado en la desintegración social: las sociedades contemporáneas son crecientemente incapaces de integrar a todos sus miembros, particularmente en el sistema económico y en los beneficios públicos y básicos, pero también en las instituciones y organizaciones formales y en las diversas redes de interacción social. La exclusión puede, por lo tanto, ser entendida como un sintoma de desintegración y una amenaza contra la cohesión social."

4 Jbídem, página 1.

" CEPAL, "La exclusión social de los grupos pobres en Chile", Santiago, Chile, 26 de junio de 1998, página 4.

6. Ibidem. 
Este estudio nos da luces sobre ciertos rasgos del fenómeno de la exclusión social, relacionándolo con el proceso de desintegración social y el debilitamiento de los lazos sociales. Pero al momento de analizar en más profundidad la realidad chilena, el estudio se limita a citar una serie de situaciones de precariedad y privación, como el desempleo, el empleo precario, el empleo parcial, las condiciones de trabajo poco adecuadas, ausencia de filiación y cotización provisional, baja productividad de las actividades, bajos niveles de ingresos, la falta de acceso a crédito, falta de acceso a la justicia y programas sociales, dificultades de participación y la exclusión sociocultural, entre otras. ${ }^{7}$ Sin embargo, hace falta un análisis más profundo de cómo el debilitamiento de los lazos sociales y la desintegración social lleva a estas situaciones de privación, en este sentido el proceso mismo de exclusión social que produce la pobreza. Es necesario diferenciar entre la exclusión como privación en sí misma, y el proceso mismo de exclusión que lleva a la privación, donde es necesario poner mayor énfasis.

Justamente es Amartya Sen quien hace estas distinciones, analizando cuál es el aporte particular de este concepto, y distinguiendo diferentes tipos de exclusión social. Para comenzar, veamos cuál es el aporte de este concepto según Sen: "El aporte del enfoque de la exclusión social no descansa en su novedad conceptual, sino mas bien en su influencia práctica de enfatizar -y centrar su atención en-el rol de las características relacionales de la privación. ${ }^{18}$ En este sentido, más que relevar solamente la existencia de dimensiones sociales de la exclusión, este concepto centra su atención en analizar las características relacionales de la privación. Y aquí es necesario hacer dos distinciones. ${ }^{9}$ De acuerdo con Sen, por un lado existen exclusiones constitutivas, que son privaciones en sí mismas, como el no poder participar libremente en una comunidad. Esta es una exclusión en sí misma, al ser una necesidad humana vital, y una expresión de su dimensión gregaria. Pero por otro lado, existen exclusiones instrumentales, o sea ciertas relaciones

Ibídcm, páginas 13-50.

* Sen, Amartya, Op. Cit., página 8.

"Ibidem, página 5. 
sociales que no son en sí privaciones fundamentales, pero que llevan o pueden llevar a otras privaciones insospechadas y aun mayores. Por ejemplo, el no tener acceso a crédito por su uso monopolizado por parte de una facción política en una comunidad campesina, aparentemente no es una privación en sí misma, pero puede llevar a importantes consecuencias a lo largo del tiempo, tales como un proceso de empobrecimiento profundo e incluso el hambre.

$\mathrm{Al}$ aplicar este concepto, se debería analizar las relaciones sociales y el proceso mismo en que aquella facción pasa a monopolizar el acceso al crédito, tanto para comprender la naturaleza multidimensional de la privación misma, como para actuar en su superación. Por ejemplo, investigar qué tipo de relaciones sociales existen entre los múltiples actores sociales al nivel local; qué actores externos intervienen al nivel local, modificando las interacciones normales; y qué lógicas socioculturales llevan a ese tipo de relaciones sociales.

\section{Enfoque de Interfaz Sociocultural}

Un enfoque que complementa este entendimiento del concepto de exclusión social es el enfoque de interfaz, acuñado por Norman Long y aplicado también por Bryan Roberts. Una interfaz es el área de intercambio entre dos o más subsistemas, "que más exactamente son subsistemas interconectados por sus interfaces para formar un solo sistema sociocultural total que corresponde a la sociedad humana en un territorio determinado. ${ }^{\prime \prime 0} \mathrm{De}$ acuerdo con este enfoque, en el nivel microlocal y regional interactúan una serie de actores, individuales o colectivos, representando diferentes cosmovisiones y miradas de mundo. Este enfoque permite analizar las diversas lógicas culturales y de conocimiento de los actores que interactúan en esos niveles, los tipos de relaciones sociales que establecen entre sí producto de esas lógicas, y las consecuencias que ese proceso tiene. De acuerdo con Norman Long:

1w Durston, John; Duhart, Daniel; Miranda, Francisca, y Monzó, Evelyn, Comunidades campesinas, agencias priblicas y clientelismos politicos en Chile, LOM Ediciones/GIA, Santiago, septiembre 2005, página 18. 
"... La noción de interfaz social se torna relevante como una forma de explorar y entender problemáticas de heterogeneidad social, diversidad cultural y los conflictos inherentes a los procesos que involucran la intervención externa. Las interfaces surgen normalmente en puntos donde diferentes, y generalmente conflictivos, mundos de vida o campos sociales intersectan, o más concretamente, en situaciones sociales o 'arenas' en las cuales las interacciones se orientan en torno a problemas de conexión, concertación, segregación, y competencia entre puntos de vista sociales, evaluativos y cognitivos. El análisis de la interfaz social apunta a elucidar los tipos y fuentes de discontinuidad y eslabonamiento social presentes en tales situaciones, y a identificar los medios organizacionales y culturales para su reproducción o transformación. También puede ayudar a desarrollar un análisis más adecuado de los procesos de transformación en politicas, ya que nos permite entender con mayor profundidad las respuestas diferenciales de grupos locales (tanto población objetivo como no-objetivo) a la intervención planificada. "II

Podemos afirmar junto a Bryan Roberts que en el contexto latinoamericano los mismos cambios sufridos por el Estado en los últimos treinta ańos han venido a multiplicar las situaciones de interfaz en el nivel local. En contraste con periodos anteriores de su historia reciente, el escenario actual en el que se desenvuelven las políticas sociales y de desarrollo en América Latina presenta ciertas características particulares que enfatizan la importancia de considerar con seriedad el aporte del concepto de exclusión social, así como este enfoque complementario de interfaz. De acuerdo con Roberts, tales rasgos se pueden resumir en tres procesos simultáneos desplegados en las últimas dos décadas: la reforma del Estado y la austeridad fiscal producto de la crisis de la deuda; el proceso de descentralización y el rol renovado del gobicrno local en la política social; y el proceso de democratización y transición a la democracia desarrollado en los distintos niveles institucionales, especialmente en el ámbito del municipio. Este triple proceso ha desembocado en una proliferación de actores en la aplicación y ejecución de las

"Long, Norman Development Sociology: Actor Perspectives. Routledge, New York and London. 2001, páginas 65 y 66, craducción librc. 
políticas sociales, los cuales se encuentran y desencuentran en el espacio micro-local, local y regional, con diversas consecuencias para las poblaciones sociales y "excluidas". Refiriéndose a los cambios en la política social en América Latina, Roberts plantea:

"La democratización ba incrementado dramáticamente el rol del gobierno local en la politica social. Mientras que en los 1960 y los 1970, el gobierno municipal estaba usualmente ausente en el campo de la politica social, sin la responsabilidad de la salud, educación o asistencia social, para los 1990, los gobiernos municipales se han convertido en los principales protagonistas en la administración de estas áreas de política social. La austeridad fiscal trae consigo dos conjuntos más de actores en politica social: el sector privado a través de asociaciones públicas-privadas y las organizaciones locales comunitarias. Tales actores se transforman en estrategias para hacer distribuir escasos fondos públicos en servicios tales como capacitación para el empleo o cuidado comunitario de los niños o para personas de tercera edad."12

Este rol renovado del municipio, en un contexto de redemocratización y reintroducción del sistema de partidos políticos, deja la política social en manos de grupos de interés político que pueden utilizar los escasos fondos públicos para fines electorales. Por otro lado, abre el espacio micro-local y regional a la emergencia de nuevos movimientos sociales, que intentarán contrarrestar la fuerza de estos grupos políticos locales, y la de otros movimientos, y aumentar su participación en el proceso de toma de decisiones. Sin embargo, este escenario eminentemente competitivo tendrá importantes consecuencias para las comunidades locales y las posibilidades para una verdadera ciudadanía, como veremos más adelante.

Volviendo a Norman Long, el análisis de interfaz ayuda también a deconstruir el concepto de intervención planificada, visualizándolo como un proceso continuo de construcción social, negociación y consulta entre diversos actores, y no simplemente la aplicación de un ya especificado plan de

\footnotetext{
Bryan Roberts, "Las nuevas políticas sociales en América Latina y el desarrollo de ciudadanía: una perspectiva de interfaz", documento presentado para cl taller "Agencia, conocimiento y poder: nucvas direcciones", Universidad de Wagenigen, Holanda, diciembre 2001, página 8.
} 
acción con productos esperados. ${ }^{13}$ Bajo este enfoque, el conocimiento, y su control o facilitación, tiene un lugar central. De acuerdo con Long, el conocimiento asume una especial significancia al abarcar el intercambio (colaboración) o conflicto entre formas de conocimiento, creencias y valores "expertas" versus otras "informales", y luchas sobre su legitimización, segregación y comunicación. ${ }^{14}$ Este énfasis tiene gran relevancia para nuestro análisis de los aportes del concepto de exclusión social, para entender la naturaleza de la privación al nivel local, los tipos de relación social que los actores establecen entre sí, y el proceso por medio del cual un grupo o actor excluye a otros. Este interés se conecta con la preocupación por las prácticas de intervención que imprime el enfoque de interfaz, centrándose en las formas emergentes de interacción, procedimientos, estrategias prácticas, tipos de discurso y categorías culturales de los diversos actores presentes en contextos específicos, ayudando en el análisis y evaluación de las consecuencias que éstas tienen para el proceso tanto de exclusión como de construcción de ciudadanía. Podemos apreciar en el siguiente Cuadro una expresión gráfica de un ejemplo de interfaces socioculturales al nivel local en Chile:

Figura $N^{\circ}$ 1: Enfoque de Interfaz

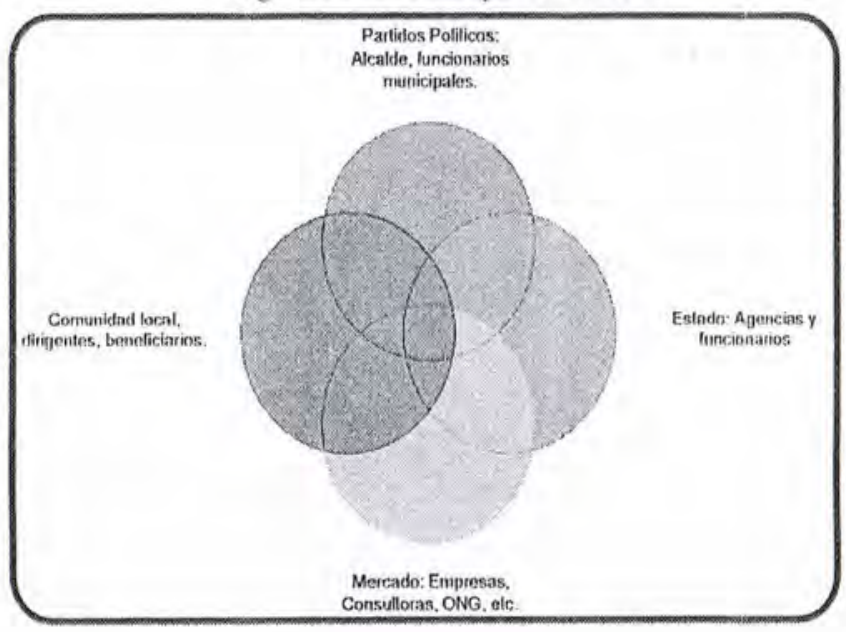

1) Long, Norman, "The Multiple Optic of Interface Analysis", UNESCO Background Paper on Interface Analysis, October 1999.

if Ibidem. 
Tal como decíamos, el análisis de interfaz permite centrarse en los tipos de vínculos que se establecen entre actores en el nivel local, analizando las relaciones sociales existentes y el nivel de exclusión social. Este análisis no solo enfatiza la causa o naturaleza de la privación, sino que también permite visualizar líneas de acción que generalmente no son consideradas al momento de enfrentar la pobreza, que tienen que ver más con las dimensiones conductuales y actitudinales de los actores sociales. Bryan Roberts identifica seis posibles interacciones entre actores en la interfaz, una tipología que permite evaluar situaciones viciosas o virtuosas, que van desde la exclusión hasta la complementariedad, ayudando en el diseño de estrategias para su superación o potenciación: ${ }^{15}$

1. Excluyente, que estigmatiza a los beneficiarios como dependientes o incompetentes (focalización individual: destruye lazos comunitarios)

2. Clientelismo partidario que premia pasividad y el voto con entrega de productos y servicios (manipulación de la focalización)

3. Competencia, que sigue principios de mercado por austeridad fiscal 4. Tecnocracia burocrática racional, que impone fórmulas

5. Encajamiento (incrustación/embededness) de servicios públicos en vínculos socio-emocionales entre agente y comunidad

6. Complementariedad o sinergia

Un elemento común a todas estas relaciones, y que está presenta en todas las situaciones de interfaz, es la dimensión de poder. En un extremo vemos actores que poseen el control sobre el uso de recursos públicos, o que representan formas profesionales de conocimiento, se imponen y dominan a los actores locales, sin recursos, pobres materialmente, y poseedores de conocimiento tradicional o informal. En otro extremo las comunidades locales presentan un alto nivel de autoestima y confianza, viviendo un proceso de empoderamiento, en colaboración con otros actores, y actuando en sinergia con el Estado. El poder está presente en ambas situaciones, pero de distintas

15 Bryan Roberts, Op. Cir., páginas I1-15. 
maneras. ¿Pero qué es poder, finalmente entonces, si está presente tanto en relaciones de dominación como de colaboración? La comprensión de este concepto es esencial para nuestro análisis de las relaciones sociales de exclusión, y su transformación en relaciones de reciprocidad y colaboración.

\section{¿Qué es Poder?}

El debate sobre la noción poder se remonta a los inicios de las ciencias sociales modernas, donde autores clásicos como Max Weber y Karl Marx, y otros posteriores, como Foucault, han realizado definiciones clásicas que perduran hasta hoy en día. Podemos decir que predomina un discurso académico y público sobre poder que lo define como la capacidad de controlar e influenciar a otros e imponerles la propia voluntad. Esto puede ser tanto a nivel personal como grupal o social, como es la relación entre clases sociales. Pero en el párrafo anterior, en que observamos no sólo relaciones de dominación, sino que también de colaboración, esta definición de poder, acuñada en un contexto histórico y cultural particular (construcción del Estado moderno y expansión mundial del capitalismo), queda limitada. A nuestro juicio, es necesario reflexionar e indagar con más profundidad.

Un interesante trabajo realizado por el antropólogo Michael Karlberg ${ }^{16}$ presenta una valiosa contribución a esta reflexión. De acuerdo con Karlberg, el concepto de poder debe diferenciarse del contexto sociocultural en el que se expresa, separando en su análisis las dimensiones distributivas y relacionales de poder. Para Karlberg, la noción contemporánea de poder responde a un contexto sociocultural moderno que él llama la cultura de la competencia (culture of contest), que imprime todas las dimensiones del modelo de sociedad occidental que se ha expandido por el mundo. En su estudio, analiza cómo ciertas construcciones discursivas hegemónicas sobre poder se han establecido como el sentido común, un proceso de naturalización histórica en que ya no se reflexiona sobre el poder porque se considera que siempre ha sido así, la capacidad de controlar y dominar a otros. Este adversarialismo normativo, como él lo define, abarcaría el funcionamiento tanto de los sis-

16. Karlberg, Michael, Begond the culture of contest, George Ronald, Oxford, 2004. 
temas económicos como los políticos y jurídicos, estableciendo al conflicto, y su expresión institucional en la competencia, como el único método de interacción entre los actores. Pero según Karlberg, esta noción niega todas las experiencias de mutualismo y cooperación del pasado, así como las del presente, $y$ sus desarrollos teóricos. ${ }^{17}$

Este discurso sobre el poder, dentro del contexto de la cultura de la competencia, se centraría únicamente en las relaciones conflictivas entre las personas, definiendo al poder inherentemente como competitivo, expresado en el control de unos sobre otros. Para entender esta cultura de la competencia, Karlberg plantea que se deben analizar las maneras en que las personas piensan y hablan sobre el poder. Como la forma en que pensamos y hablamos influye en nuestras conductas, un discurso centrado solamente en las dimensiones conflictivas del poder tendría significativas implicancias sociales, tales como la misma exclusión social. En este sentido, conectado este análisis con los aportes del concepto de exclusión social, debemos analizar el contexto sociocultural en que las relaciones de poder se establecen, esto es los códigos culturales expresados en sistemas de representación y discursos, separándolos del concepto mismo de poder. En la sociedad contemporánea, al centrar la atención en las relaciones conflictivas y competitivas de poder únicamente, los aspectos mutualistas desaparecen, y se prescriben modelos de organización e interacción sociales que responden a esa noción únicamente, surgiendo relaciones sociales que profundizan la exclusión. ${ }^{18} \mathrm{Y}$ un sistema social construido sobre la base de la cultura de la competencia es inherentemente excluyente, siendo posible su superación solamente a través de su propia transformación.

El último Informe de Desarrollo Humano en Chile del PNUD, titulado "El Poder: para qué y para quién", realiza una valiosa contribución en esta reflexión. En uno de sus capítulos, en que analiza los imaginarios y discursos sobre poder de diversos grupos sociales del país, podemos apreciar la presencia de esta llamada "cultura de la competencia" y su influencia en su

17 Ibídem, páginas 36-79.

18 Ibídem, páginas 1-19. 
noción de poder. ${ }^{19}$ Expresiones como "su autoridad, uno no es nada en comparación con ellos", "no quiero que nadie me mande", "la clase alta siempre ha dominado", "a veces la misma sociedad lo para", "siempre está la piedra de tope", "un proyecto-país, manejado por cúpulas, por el poder", "cuando uno va a la oficina a pedir algo... hay que como bumillarse", "en todas partes lo pasan a llevar a uno", "eso es lo que hace mucha gente, que en parte no lucha por sus derechos", "la gente está como entre la espada y la pared", "inhibido por una cultura que fomenta la subordinación", "generalmente nos gusta dominar al otro, en todo sentido", entre otras, reflejan una noción de poder definido por el control de unos sobre otros únicamente, y relaciones sociales caracterizadas por la competencia.

No se trata de negar la existencia de esas realidades caracterizadas por la dominación, al contrario, la idea es transformarlas. Más bien, lo que se trata es generar una noción de poder más amplia, y que considere una gama mayor de relaciones sociales, pues como hemos mencionado, una noción de poder centrada únicamente en el conflicto genera formas de organización e interacción centradas únicamente en esa dimensión, como ocurre en los sistemas políticos, económicos y jurídicos analizados por Karlberg. La misma frustración y statu quo que produce el sistema de partidos políticos moderno, por ejemplo, es producto de una concepción competitiva y adversaria del poder y de la democracia; las relaciones clientelares que prolongan este sistema al nivel de las bases, produce más exclusión y erosión del capital social que un aumento del bienestar integral de la población. ${ }^{20}$ En el mismo informe del PNUD, se presentan otras expresiones discursivas que reflejan la necesidad de darle un espacio también a las dimensiones más mutualistas del poder: "pero hay otro alcance del término 'proyecto', tiene que

19 PNUD, Informe de Desarrollo Humano en Chile. El poder: ¿para qué y para quién?, Santiago de Chile, dicicmbre de 2004, páginas 94-114.

3n Duhart, Danicl, "Programas sociales, partidos políticos y liderazgos familiares: crosión del capital social en una comunidad mapuche", en Capital social: potencialidades analiticas y metodológicas para la superación de la pobreza, CEPAL, División de Desarrollo Social, Serie Seminarios y Conferencias No31, Santiago, ocuubre 2003; Auyero, Javier. "Evita como performance. Mediación y resolución de problenas entre los pobres urbanos del Gran Buenos Aires", en ¿¿Favores por votos? Estudios sobre clientelismo politico contemporáneo, Editorial Losada, Buenos Aires, 1997. 
ver con una cuestión más social, más de comunidad, de pais", "aquí se propicia el trabajo en equipo, que hay relaciones más o menos horizontales", "Yo funciono más con adhesión que con órdenes." 21

En esta misma línea, Michael Karlberg se refiere a la emergencia de una noción alternativa de poder, que es el poder 'para'. Esta concepción, diferente a la noción clásica de poder 'contra' (dominación, conflicto), define al poder como una capacidad, por ejemplo, el ser capaz de realizar una acción o entablar una relación. En este sentido, el control de unos sobre otros podría ser un tipo de relación que es posible establecer en el uso de esta capacidad, pero no la única. También sería posible establecer relaciones mutualistas de cooperación y colaboración, que no son excluyentes, por medio de esta capacidad. Aquí nos acercamos a la noción de poder 'con' otros, que resalta la dimensión cooperativa y no conflictiva del poder. ${ }^{22}$ Para el mismo Anthony Giddens el poder es una "capacidad transformadora" o la "capacidad de lograr resultados". ${ }^{23}$

De este modo, podemos decir que, estrictamente hablando, la noción de poder se refiere a una capacidad o fuerza para llevar a cabo una acción, sea esta adversaria o mutualista. En este sentido, la noción de poder es independiente de la relación social que se establece con su uso. Esta distinción es clave al momento de analizar el fenómeno de la exclusión social, e indagar en formas para su superación. Esta separación entre el contexto sociocultural, que puede privilegiar cierto tipo de relaciones sociales por sobre otras, es crucial, porque implica que para superar ciertas situaciones cxtendidas de exclusión social, más que integrar a los excluidos al sistema imperante, es necesaria una transformación de la matriz sociocultural que imprime o privilegia ese tipo de relaciones sociales. El cambio está en el nivel de las actitudes y conductas, así como los códigos culturales y sistemas de representación que privilegian ciertas construcciones discursivas por so-

21 PNUD. Op. Cit.

22 Karlberg, Michael, Op. Cit., páginas 25-28.

23 Giddens, Anthony, Constitution of Society, páginas 15 y 157, citado en: Karlberg, Michael, Beyond the culture of contest, Gcorge Ronald, Oxford, 2004, página 28. 
bre otras. Este análisis amplía nuestra discusión sobre el concepto de exclusión social a una dimensión bastante más abierta, ubicándola en el nivel del análisis sociocultural y de las relaciones sociales.

Karlberg no sólo hace una distinción entre la definición de poder como capacidad y las relaciones sociales con que puede ser desarrollado, sino que además las distingue de la noción de distribución de poder. Una situación en que se presenta una distribución desigual de poder puede tener un destino muy diferente dependiendo de si se presenta bajo una relación adversaria o mutualista de poder. Si se expresa en el interior de una cultura adversaria, claramente llevará a una situación de dominación, profundizando la desigualdad. Pero por otro lado, si se presenta en un contexto de cultura mutualista, puede llevar más bien a una relación de empoderamiento asistido, evolucionando finalmente a un balance de poder. Esta diferenciación es extremadamente importante para el estudio de la exclusión, pues entrega ciertas luces hacia dónde se puede avanzar en el proceso de transformación sociocultural sistémica. Karlberg desarrolla un modelo que distingue estas diferentes dimensiones de poder, el cual se puede reflejar en el siguiente esquema:

\section{Tabla No1: Poder como Capacidad ${ }^{24}$}

\begin{tabular}{|c|c|c|c|}
\hline \multicolumn{2}{|c|}{$\begin{array}{c}\text { Relaciones adversarias } \\
\text { "poder contra" } \\
\text { Competencia }\end{array}$} & \multicolumn{2}{|c|}{$\begin{array}{l}\text { Relaciones mutuales } \\
\text { "poder con" } \\
\text { Cooperación }\end{array}$} \\
\hline $\begin{array}{l}\text { Inequidad } \\
\text { "poder sobre" }\end{array}$ & $\begin{array}{c}\text { Equidad } \\
\text { "balance de poder" }\end{array}$ & $\begin{array}{l}\text { Inequidad } \\
\text { "empoderamiento } \\
\text { asistido" }\end{array}$ & $\begin{array}{c}\text { Equidad } \\
\text { "cmpoderamiento } \\
\text { mutuo" }\end{array}$ \\
\hline $\begin{array}{l}\text { Coerción } \\
\text { Dominación } \\
\text { Opresión }\end{array}$ & $\begin{array}{l}\text { "Tabla" } \\
\text { Compromiso } \\
\text { Frustración }\end{array}$ & $\begin{array}{l}\text { Educación } \\
\text { Nutrición } \\
\text { Asistencia }\end{array}$ & $\begin{array}{c}\text { Sinergia } \\
\text { Colaboración } \\
\text { Coordinación }\end{array}$ \\
\hline Ganar/perder & Perder/perder & (Ganar)/ganar & Ganar/ganar \\
\hline
\end{tabular}

${ }^{24}$ Karlberg, Michael, Op. Cit., página 30. 


\section{Revista MAD "Nuevas exclusiones en la complejidad social contemporánea"}

Al mirar el cuadro, podemos apreciar que la definición clásica de poder como control, en este caso 'poder sobre', se reduce a sólo una cuarta parte de las posibilidades, demostrando cuan estrecha y limitada es aquella noción. Además, podemos observar que aunque exista una igualdad o balance de poder en la sociedad, si ésta es aplicada por medio de relaciones adversarias o de competencia, puede llevar a la profundización de esa misma desigualdad. Como ocurre en la mayoría de las democracias representativas y partidistas, la competencia entre grupos con similar nivel de poder lleva más bien a la apatía y la frustración, y no al logro de transformaciones valiosas para una mayoría. Por otro lado, aunque exista una relación temporal de desigualdad de poder, su desarrollo a través de relaciones de cooperación o mutuales, favorecen una situación de ganar/ganar, donde es posible avanzar desde una relación de empoderamiento 'asistido' hacia una de empoderamiento 'mutuo', similar a la escala desarrollada por Bryan Roberts, donde el tipo ideal es la sinergia y la colaboración.

De este modo, la diferenciación entre poder mismo, como una capacidad o fuerza, el tipo de relación social y su distribución social, es esencial para nuestro análisis de la exclusión social, así como para la búsqueda de salidas, como veremos a continuación.

\section{Relaciones Sociales, Cultura y Transformación}

Tal como decíamos, el análisis de la exclusión social y el poder en el contexto mayor de una 'cultura de la competencia' nos lleva a reflexionar sobre la necesidad de políticas de integración que impliquen una nueva reintegración social, o una transformación misma del sistema. La competencia y el conflicto son inherentemente excluyentes, y si son definidas como los principios gobernantes de un sistema social, es imposible su superación. No se trata en este análisis de negar el conflicto y las diferencias entre individuos y grupos humanos, más bien nos referimos a no convertirlas en un supuesto de la naturaleza humana, y a organizar a la sociedad y sus diversos sistemas (económico, político, jurídico, etc.) alrededor de ella. Debe haber instrumentos e instituciones para mediar en momentos de conflicto, pero en el marco de una cultura mutualista éstas adquieren otro sentido. Ade- 
más, este análisis nos ayuda a enfocar las propuestas de salida no en el concepto mismo de poder, sino más bien en las relaciones sociales y el cambio cultural de los diversos actores en la interfaz. El estado opuesto a la exclusión es el de ciudadanía, pero no una noción formal de ciudadanía, sino más bien una centrada en la capacidad constituirse en un actor social, construyendo relaciones mutuales de poder con otros actores, contribuyendo de este modo a un cambio cultural y así como a una distribución más equitativa del poder en la sociedad.

Una línea interesante en esta lógica es la propuesta del aprendizaje para la transformación social. De acuerdo con el Institute of Development Studies (IDS) de la Universidad de Sussex, Inglaterra:

"El cambio social requiere que individuos, solos y colectivamente, lleven a cabo cambios de comprensión sobre lo que es posible en la formación de la condición humana. La educación, cuando es basada en ciclos continuos de reflexión y acción, y fundada tanto en la teoría como en la práctica, puede apoyar profundos y transformativos procesos de aprendizaje y resultados, llevando potencialmente a cambios en un más amplio nivel organizacional y societal. La participación, por medio de un rango de innovaciones y prácticas exitosas, es percibida en tener el potencial para influir en un cambio social favorable a los pobres y contribuir a la reducción de la pobreza y a una mayor justicia social al fortalecer los derechos ciudadanos y su voz, lo cual a su vez puede influenciar el diseño de politicas, facilitar la gobernabilidad local, y mejorar el control social y responsabilidad de las instituciones. Pero la participación significativa requiere aprendizaje, cambios en la conducta, actitudes y relaciones de poder. ¿Cuál es la relación entre educación, participación y cambio social? ¿Qué necesita ser aprendido para lograr tanto la transformación de los individuos como de la sociedad? ¿Y dónde y con qué medios debe tal aprendizaje ser convenido?"25

55 Stackpool-Moore, Lucy; Taylor, Peter; Pettit, Jethro, y Millican, Juliet (editores), Currents of Change. Exploring relationships between teaching. learning and development, Institute of Development Studies, Susscx, 2006, página 3, traducción libre. 
Esta iniciativa pone el acento en la transformación de actitudes y conductas de diversos actores sociales, para la generación de un marco cultural más favorable a la superación de la desigualdad. De este modo, relaciones sociales excluyentes, que son las que priman en el sistema actual, se transformarían en relaciones de colaboración y de empoderamiento asistido, para luego avanzar hacia el empoderamiento mutuo. El desarrollo de capacidades es parte de un proceso de aprendizaje, donde no sólo entran en juego ciertos conceptos, sino que también actitudes, destrezas y cualidades. En una sociedad acostumbrada a una noción de poder como coerción, y a su ejercicio por medio de relaciones adversarias o de competencia, el desarrollo de un modelo alternativo de ciudadanía y poder requiere un proceso de cambio cultural profundo.

Una imagen que es útil para ilustrar este proceso es visualizar las actitudes y conductas de la cultura de la competencia como un modelo mental estático, inconsciente e inconsistente, en el cual se presentan discursos ligados al poder, pero prácticas contradictorias que más que transformar la realidad, profundizan ciertas relaciones de exclusión. Por medio de un proceso de capacitación, reflexión y acción permanentes, gradualmente se transforman estos modelos mentales estáticos en marcos conceptuales conscientes, consistentes y evolutivos, al ir aprendiendo y aplicando cada vez mejor las capacidades de un modelo alternativo de ciudadanía. El siguiente esquema ilustra este proceso:

Figura No2: Transformando modelos mentales en marcos conceptuales ${ }^{26}$

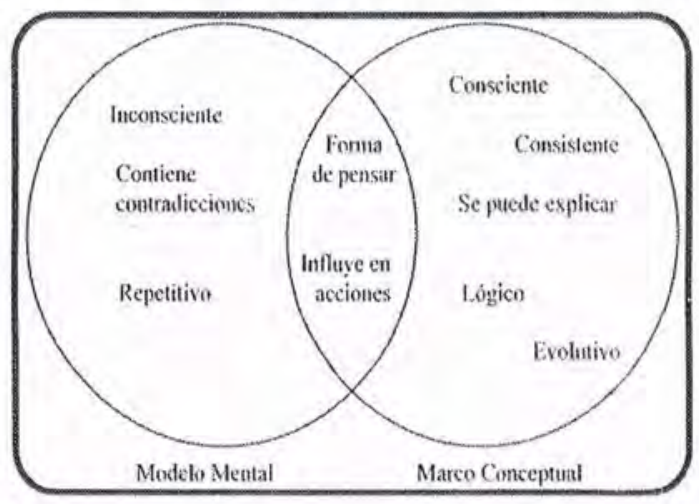

26. Hernández, Juanita y Anello, Eloy, Capacitación de agentes de desarrollo comunitario, Módulo No2,

"Conceptos de Aprendizaje y Desarrollo", Universidad Nur, Instituro Superior de Educación Rural, Santa Cruz de la Sierra. 
El concepto de transformación sistémica que acuñamos no es un concepto que se reduce a la transformación de la estructura social únicamente. La transformación de estructuras se refiere tanto a las estructuras sociales como a las mentales, culturales, e institucionales, respondiendo a las dimensiones psicoestructurales y socioestructurales de la cultura ${ }^{27}$. En este sentido, el modelo de transformación de modelos mentales estáticos en marcos conceptuales coherentes y concientes, se traduce en la práctica en un proceso simultáneo de transformación personal y social, por medio del desarrollo y aplicación de capacidades (reflejado en conceptos, actitudes, conductas $y$ destrezas).

No es nuestra intención entrar en un análisis más profundo de esta última temática, tarea que será parte de otro artículo sobre ciudadanía que se encuentra en elaboración. Sólo deseamos mencionar que existen una serie de propuestas innovativas y experiencias muy interesantes en América Latina, y otros continentes, sobre formas alternativas de ciudadanía, que aportan al mencionado proceso de cambio cultural necesario para la superación de la exclusión social, transformando a la vez el sistema social. ${ }^{28}$ Haremos referencia a solo un caso, cual es el paradigma del Liderazgo Transformacional desarrollado por el Instituto Superior de Educación Rural de la Universidad Nur, en Santa Cruz, Bolivia.

Este modelo presenta una noción de liderazgo comunitario o colectivo, dirigido a la transformación personal y social, y centrado en el desarrollo de capacidades. Las capacidades se dividen en tres tipos: para la transformación personal, para la transformación de relaciones interpersonales, y para la transformación social. Algunas capacidades son las siguientes: ${ }^{29}$

27 Karlberg, Michacl, Op. Cit., páginas 5-7.

2* Resseau Cultures, Cultures ơ Developmenu, No35/36, Journal of the South-North Network Cultures and Development, Special Issue: "The Spirituality of Social Commitment", Brussels, november 1999; Correa, Gustavo y Torné de Valcárcel, Francia, Para salvar las barreras. El Sistema de Aprendizaje Titorial - SAT, FUNDAEC, Cali, encro de 1995; Chambers, Robert, Who's Reality Coumts? Putting the furst last, Intermediate Technology Publications, London, 1997.

2n Hernández, Juanita, y Ancllo, Eloy, Liderazgo Moral. Capacitación de maestros rurales, Módulo Nol, Universidad Nur, Instituto Superior de Educación Rural, Santa Cruz de la Sierra, 1993. 
Revista MAD "Nucvas exclusiones en la complejidad social contemporánea"

\section{Capacidades para la transformación personal:}

- Capacidad de evaluar fortalezas y debilidades: la autoevaluación.

- Capacidad de aprender de la reflexión sistemática sobre la acción dentro de un marco conceptual consistente y evolutivo.

- Capacidad de tomar iniciativa de manera creativa y disciplinada.

- Capacidad de pensar sistémicamente en la búsqueda de soluciones.

Capacidades para la transformación de las relaciones interpersonales:

- Capacidad de alentar a otros y de trabajar en equipo.

- Capacidad de participar en la consulta de toma de decisiones colectivas.

- Capacidad de ser un miembro responsable del grupo familiar y la comunidad.

- Capacidad de promover y construir unidad en diversidad.

Capacidades que contribuyen a la transformación social:

- Capacidad de crear una visión de un futuro deseado basada en principios.

- Capacidad de comprender las relaciones de dominación y de contribuir a su transformación en relaciones basadas en la reciprocidad, la cooperación y el servicio mutuo.

- Capacidad de comprometerse con el proceso de potenciar actividades educativas.

Las implicancias y supuestos de este modelo para nuestra discusión sobre la exclusión son muy interesantes. Su énfasis en la dimensión relacional del liderazgo conecta con el aporte de este concepto, así como con la necesidad de transformar relaciones sociales adversarias hacia un marco cultural más mutualista. La noción de capacidades nos conecta con nuestra definición de poder como capacidad, su distribución equitativa y su aplicación a través de cierto tipo de relaciones sociales. Además, su énfasis en la transformación, tanto personal, como interpersonal y social, contribuye claramente al proceso de cambio sociocultural que implica la superación de la exclusión social, transformando el sistema social gobernado por la cultura 
de la competencia en uno más amplio que da lugar a sistemas de organización económica y política más colaborativos. Esta transformación sistémica se expresa tanto en las estructuras sociales e institucionales, como las mentales y culturales, y su estrecha interrelación.

\section{Palabras Finales}

De este modo, hemos reflexionado sobre las implicancias y alcances del concepto de exclusión social, lo cual nos ha llevado a un análisis muy profundo de diversos temas. Iniciamos esta reflexión preguntándonos por los aportes de este concepto, y las implicancias para el análisis de la privación. Después, entramos a reflexionar sobre las implicancias más amplias de este concepto, al nivel de los modelos de desarrollo, y concluimos que su análisis implica no la mera inclusión de sectores excluidos al sistema, sino que más bien la transformación del mismo sistema, inherentemente competitivo.

Un concepto clave en este proceso fue el de poder, el cual aplicado con un enfoque de interfaz permite cualificar los tipos de vínculos que se establecen entre los diversos actores sociales en un espacio micro-local y regional. Analizamos las definiciones tradicionales de poder, y llegamos a la conclusión de que son producto de un particular contexto histórico y cultural, no siendo satisfactoria su aplicación actual. Para eso, llegamos a una definición de poder como capacidad, diferenciándola de las relaciones sociales con que es aplicado, adversarias o mutualistas, y su distribución en la sociedad. De este modo llegamos a dos tipos generales de poder: el 'poder con' y el 'poder contra', dando lugar a una gama más amplia de situaciones, facilitando a la vez la búsqueda de salidas a situaciones de exclusión social.

De esta forma llegamos al análisis de la propuesta del aprendizaje para la transformación social, que centra su análisis en las dimensiones socioculturales del desarrollo y la necesidad de transformar las actitudes y conductas que gobiernan las relaciones sociales entre los agentes o actores sociales. De este modo sería posible contribuir a un proceso de transformación sociocultural mayor, superando la exclusión social a través de la transformación misma del sistema. Otras propuestas en esta línea serán partes de otro artículo en elaboración, centrado en la noción de ciudadanía como desarrollo de capacidades. 


\section{Bibliografía}

AuYero, JAVIER. 1997. "Evita como performance. Mediación y resolución de problemas entre los pobres urbanos del Gran Buenos Aires", en ¿Favores por votos? Estudios sobre clientelismo politico contemporáneo, Editorial Losada, Buenos Aires.

BENGOA, José. 1996. "La exclusión", en La comunidad perdida, Ediciones Sur, Santiago.

CEPAL. 1998. La exclusión social de los grupos pobres en Chile. Santiago.

Chambers, Robert. 1997. Who's Reality Counts? Putting the first last, Intermediate Technology Publications, London.

Correa, Gustavo y Francia Torné de Valcorcel. 1995. Para salvar las barreras. El Sistema de Aprendizaje Tutorial - SAT, FUNDAEC, Cali.

Duhart, Daniel. 2003. "Programas sociales, partidos políticos y liderazgos familiares: erosión del capital social en una comunidad mapuche", en Capital social: potencialidades analiticas y metodologicas para la superación de la pobreza, CEPAL, Santiago.

Durston, John; Duhart, Daniel; Miranda, Francisca y Monzón, Evelin 2005. Comunidades campesinas, agencias públicas y clientelismos politicos en Chile, LOM Ediciones, Colección Ciencias Humanas, Santiago.

Hernandez, Juanita, y Anello, Eloy. 1993. Liderazgo Moral. Capacitación de maestros rurales, Módulo No1, Universidad Nur, Instituto Superior de Educación Rural, Santa Cruz de la Sierra.

Karlberg, Michael. 2004. Beyond the culture of contest, George Ronald, Oxford.

Long, Norman. 1999. "The Multiple Optic of Interface Analysis", UNESCO Background Paper on Interface Analysis, October.

Long, Norman. 2001. Development Sociology: Actor Perspectives. Routledge, New York and London. 
Nun, José, 1969, «Superpoblación relativa, ejército industrial de reserva y masa marginal, en Revista Latinoamericana de Sociologia, Buenos Aires, 2:174-236.

Nun, José, 2001, Marginalidad y exclusión social, Fondo de Cultura Económica, Buenos Aires, $1^{\text {a }}$ edición.

PNUD. 2004. Informe de Desarrollo Humano en Chile. El poder: ¿para quéy para quién?, Santiago de Chile.

Quijano, Anibal, 1970, Redefinición de la dependencia y proceso de marginalización en América Latina, Documento de la CEPAL, Santiago.

Resseau Cultures. 1999. Cultures \& Development, No35/36, Journal of the South-North Network Cultures and Development, Special Issue: "The Spirituality of Social Commitment", Brussels, november.

Roberts, Bryan. 2001. "Las nuevas políticas sociales en América Latina y el desarrollo de ciudadanía: una perspectiva de interfaz", documento presentado para el taller "Agencia, conocimiento y poder: nuevas direcciones", Universidad de Wagenigen, Holanda.

Sen, Amartya. 2000. Social Exclusion: concept, application and scrutiny, Office of Environment and Social Development, Asian Development Bank.

Stackpool-Moore, lucy; Taylor, Peter; Pettit, Jethro, y Mullican, JuLier (editores). 2006. Currents of Change. Exploring relationships between teaching, learning and development, Institute of Development Studies, Sussex.

Vekemans, Roger, y Silva, Ismael, 1969, "El concepto de marginalidad", en Marginalidad en América Latina: un ensayo de diagnóstico, DESAL, Herder, Barcelona. 